\title{
ANÁLISIS DE LA POSICIÓN HORIZONTAL DEL SISTEMA GALILEO EN EL ECUADOR CONTINENTAL UTILIZANDO EL SOFTWARE RTKLIB
}

\author{
HORIZONTAL POSITION ANALYSIS OF GALILEO SYSTEM IN CONTINENTAL \\ ECUADOR USING RTKLIB SOFTWARE
}

\section{DENNYS ENRÍQUEZ'; ALFONSO MORILLO $^{2}$}

${ }^{1}$ CARRERA DE INGENIERÍA GEOGRÁFICA Y DEL MEDIO AMBIENTE. UNIVERSIDAD DE LAS FUERZAS ARMADAS - ESPE. Av. General Rumiñahui s/n, Sangolquí, Ecuador. E-mail: daenriquez5@ espe.edu.ec

${ }^{2}$ INSTITUTO GEOGRÁFICO MILITAR - IGM. Siniergues y Telmo Paz y Miño s/n. Quito, Ecuador. E-mail: alfonso.morillo@mail.igm.gob.ec

Recibido: 30 de agosto de 2018 / Aceptado : 10 de diciembre de 2018

\begin{abstract}
RESUMEN
La navegación por satélite ha evolucionado de manera que se ha podido evidenciar un rápido crecimiento del Sistema de Navegación Global por Satélite (GNSS), que actualmente, está conformado por GPS, GLONASS, BeiDou, QZSS, IRNSS y Galileo. La ausencia de información del comportamiento de la constelación Galileo sobre el Ecuador continental muestra problemas, ya que no se ha identificado el error que presenta el sistema sobre la posición horizontal, esto implica el desconocimiento de las aplicaciones útiles para dicha constelación. Por lo tanto, el objetivo del presente proyecto es analizar la posición horizontal del Sistema Galileo, a partir del cálculo de la distancia entre coordenadas UTM obtenidas mediante el método PPP estático con soluciones semanales SIRGAS, para determinación del error en posición que presenta esta constelación en el Ecuador Continental. Para cumplir con esto, se rastreó la constelación mediante 3 Estaciones de Monitoreo Continuo del Ecuador pertenecientes a la REGME, enlazadas a la red SIRGAS-CON. Los datos obtenidos se procesaron en cuatro bloques con diferentes lapsos de tiempo con la técnica PPP estático en el software RTKLIB versión 2.4.2. Esto se realizó con intervalos de procesamiento de 1 y 30 segundos. Finalmente, se calculó la distancia horizontal con respecto a las soluciones semanales SIRGAS. Los resultados obtenidos, muestran que en los bloques 1 y 2 las estaciones EPEC, BHEC y CXEC presentan un error aproximado de 8, 2 y 1 metro respectivamente. El bloque 4, cuyos lapsos de tiempo están entre 1.5 y 24 horas, poseen un error por debajo de los decímetros. Como conclusión de este trabajo, se muestra que las estaciones BHEC y CXEC presentaron un error medio de 1.5 metros durante los primeros 30 minutos de rastreo, que es aplicable para diferentes actividades como la navegación o la georreferenciación de cartografía a escalas menores que 1:5000. Mientras que, la estación EPEC, presentó un error más alto que las otras dos estaciones, aproximadamente 8 metros durante los primeros minutos de rastreo, esto puede deberse a la ubicación geográfica, la geometría de los satélites, y a posibles pérdidas de señal.
\end{abstract}

Palabras clave: GNSS, PPP, SIRGAS, REGME, UTM, GLONASS, BEIDOU, GPS. 


\begin{abstract}
Satellite navigation has evolved in a way which can be evidenced by the fast growth of the Global Navigation Satellite System (GNSS). Nowadays, it is conformed by GPS, GLONASS, BeiDou, QZSS, IRNSS, and Galileo. The absence of information on the behavior of the Galileo constellation on continental Ecuador shows a problem; since the error that the system presents on the horizontal position has not been identified, it implies that it is unknown for which applications it would be useful. For this reason, this project has raised the objective of analyzing the horizontal position of the Galileo System; from the calculation of the distance between UTM coordinates obtained by the static PPP method with weekly solutions SIRGAS, to determine the error in position that presents this constellation in the Continental Ecuador. To accomplish this, the constellation was traced through 3 continuous Monitoring Stations of Ecuador belonging to the REGME, linked to the SIRGAS-CON network, where data obtained were processed in four blocks with different time periods with the static PPP technique using the software RTKLIB version 2.4.2, this was carried with processing intervals of 1 and 30 seconds. Finally, the horizontal distance was calculated with respect to the weekly SIRGAS solutions. The results obtained show that in blocks 1 and 2 the EPEC, BHEC, and CXEC stations present an approximate error of 8, 2, and 1 meter, respectively. For Block 4, its time lapses are between 1.5 and 24 hours, having an error below the decimeters. As a conclusion of this work, it is presented that the BHEC and CXEC stations showed an average error of 1.5 meters during the first 30 minutes of tracking, which is applicable for different activities such as navigation or georeferencing of cartography at scales smaller than 1:5000. While, the EPEC station, showed a higher error tan the other two stations, approximately 8 meters during the first minutes of tracking, this may be due to the geographical location, the geometry of the satellites, and possible signal losses.
\end{abstract}

Key words: GNSS, PPP, SIRGAS, REGME, UTM, GLONASS, BEIDOU, GPS.

\title{
RESUMO
}

A navegação por satélite tem evoluído de forma que é possível evidenciar o rápido crescimento do Sistema Global de Navegação por Satélite (GNSS), que atualmente é conformado de GPS, GLONASS, BeiDou, QZSS, IRNSS y Galileo. A ausência de informação sobre o comportamento da constelação Galileo no Equador continental mostra problemas, uma vez que o erro que o sistema apresenta na posição horizontal ainda não foi identificado, isto implica o desconhecimento das aplicações úteis para dita constelação. Portanto, o objetivo do presente projeto é analisar a posição horizontal do Sistema Galileo, a partir do cálculo da distância entre coordenadas UTM obtidas pelo método PPP estático com soluções semanais SIRGAS para determinação do erro em posição que apresenta esta constelação no Equador continental. Para alcançar isto, a constelação foi rastreada através de três estações de monitoramento contínuo do Equador pertencentes à REGME, ligadas à rede SIRGAS-CON. Os dados obtidos foram processados em quatro blocos com diferentes espaços de tempo mediante a técnica PPP estática no software RTKLIB versão 2.4.2. Isto foi realizado com intervalos de processamento 1 e 30 segundos. Finalmente, calculou-se a distância horizontal em relação às soluções semanais do SIRGAS. Os resultados alcançados apresentam que no bloco 1 e 2, as estações EPEC, BHEC e CXEC tem um erro aproximado de 8,2 e 1 metro, respectivamente. No bloco 4, cujos intervalos de tempo situam-se entre 1.5 e 24 horas, contém um erro de ordem menor dos decímetros. Para concluir este trabalho, mostra-se que as estações BHEC e CXEC apresentam um erro médio de 1.5 metros durante os primeiros 30 minutos de rastreamento, que tem aplicação para várias atividades como navegação ou referência para mapeamento de diferentes escalas menores que 1:5000. Enquanto, a estação EPEC, apresentou um erro maior que as outras duas estações, aproximadamente 8 metros durante os primeiros minutos de rastreamento, isto pode ser devido à localização geográfica, a geometria dos satélites, e possíveis perdas de sinal.

Palavras chaves: GNSS, PPP, SIRGAS, REGME, UTM, GLONASS, BEIDOU, GPS. 


\section{INTRODUCCIÓN}

Décadas atrás, el sistema GPS, fue el primer sistema de posicionamiento que brindaba contribuciones al sector científico con muchas aplicaciones, principalmente al estudio de las ciencias de la tierra, así como también al área de ingeniería (Li et al., 2015). Posterior a este, se desarrolló GLONASS, el sistema de posicionamiento Ruso, que similar a GPS se crearon con fines militares y las aplicaciones civiles eran muy limitadas (Huerta et al., 2005).

La navegación por satélite ha evolucionado de manera que se ha podido evidenciarun rápido crecimiento del Sistema de Navegación Global por Satélite (GNSS), que actualmente, está conformado por GPS, GLONASS, BeiDou, QZSS, IRNSS y Galileo; las ventajas que brinda GNSS es que sus sistemas trabajan independientemente de los demás, son interoperables entre sí y cada constelación transmitirá tres frecuencias, es por lo que se lo considera multi-constelación y multi-frecuencia. Realizar un posicionamiento GNSS trae beneficios para la calidad del punto rastreado a través del incremento significativo de la visibilidad de los satélites, la reducción de la dilución de la precisión también ayuda a mejorar la confiabilidad para aplicaciones como el posicionamiento del punto preciso (PPP) (Montenbruck et al., 2017).

Galileo, es un sistema de navegación por satélite desarrollado por la Agencia Espacial Europea que entró en funcionamiento el 15 de diciembre de 2016. Se caracteriza por ser de uso civil y ofrece una amplia gama de servicios avanzados de posicionamiento. Además, la Comisión Europea, (2016), afirma que Galileo es totalmente interoperable con GPS, pero Galileo será más preciso y fiable para los usuarios finales cuando el sistema se complete en 2020.

Alrededor del mundo, se han realizado trabajos que utilizan datos de la señal del sistema Galileo, como es el caso del proyecto publicado por Li et al., (2015), quienes utilizan la técnica PPP estático para procesar datos de la constelación Galileo, el error que presenta durante las primeras 6 horas de rastreo es mayor al orden del metro y, a partir de las 18 horas de rastreo, el error es menor a 10 centímetros. Por otro lado, en el trabajo publicado por Cai et al., (2015), procesan datos de GPS mediante PPP obteniendo errores en posición de $3.9 \mathrm{~cm}, 1.6 \mathrm{~cm}$ y $5.7 \mathrm{~cm}$ para este, norte y altura respectivamente; al añadir datos de la constelación Galileo al procesamiento, no se notó un significante cambio debido al limitado número de satélites del sistema europeo.

Cogo et al., (2012), en Argentina, analizaron la Multi-constelación GNSS mediante el empleo de datos de 3 constelaciones, GPS, GLONASS y Galileo. Se determinó el error en posición utilizando 2 métodos (Steepest Descent y Quasi Newton). Los resultados muestran que, mediante el uso del primer método, Steepest Descent, se obtienen errores en posición horizontal de entre 20 - 30 metros con ninguna iteración y se puede llegar a tener errores de 5 centímetros con hasta 30 iteraciones. El método, Quasi Newton, 
muestra errores en posición horizontal con una iteración de entre 20 y 30 metros, mientras que, con 5 iteraciones, se obtiene un error centimétrico.

En el Ecuador, no se han realizado trabajos utilizando datos de la constelación Galileo. Por otro lado, existe un trabajo realizado por Albán et al., (2017), en el cual hace referencia al procesamiento de datos GPS mediante la técnica PPP estático. Los resultados obtenidos muestran que a partir de esta técnica se puede obtener errores de $1 \mathrm{~m}, 50 \mathrm{~cm}, 30 \mathrm{~cm}$ y 20 $\mathrm{cm}$, empleando $2,7,10$ y 20 minutos respectivamente.

La ausencia de información del comportamiento de la constelación Galileo sobre el Ecuador continental muestra problemas, ya que no se ha identificado el error que presenta el sistema sobre la posición horizontal, esto implica el desconocimiento de las aplicaciones útiles para dicha constelación. Por lo tanto, el objetivo del presente proyecto es analizar la posición horizontal del Sistema Galileo, a partir del cálculo de la distancia entre coordenadas UTM obtenidas mediante el método PPP estático con soluciones semanales SIRGAS, para determinación del error en posición que presenta esta constelación en el Ecuador Continental.

\section{CONSTELACIÓN GALILEO}

Galileo es el Sistema Global de Navegación por Satélite perteneciente a la Unión Europea, sistema que está bajo el control civil el cual ofrecerá servicios de posicionamiento de alta precisión con cobertura mundial (Ventura-Traveset, 2016). Todos los productos de Galileo están referidos al Marco de Referencia Terrestre de Galileo (GRTF, por sus siglas en inglés). De la realización y mantenimiento de éste está encargado el Proveedor de Servicios Geodésicos de Galileo (GGSP). El GRTF será compatible con el último ITRF con una precisión dentro de los 3 centímetros. La conexión al ITRF es realizada y validad por las estaciones de la IGS (International GNSS Service) (Gendth, 2011).

El programa Galileo será independiente del sistema GPS, pero como ventaja será complementario e interoperable con él. Además, facilitará la robustez del GNSS permitiendo el desarrollo de nuevas aplicaciones bajo la explotación de las capacidades de un sistema civil. Este sistema de navegación tendrá una cobertura global lo que permitirá proveer un mercado mundial, y una de las diferencias claves con GPS, es que Galileo permanecerá bajo el control de autoridades civiles (ESA, 2017). En la actualidad, el sistema Galileo posee en órbita 26 satélites y, se espera que para el 2020 esta constelación se complete con un total de 30 (ver figura 1) (ESA, 2018).

El sistema Galileo consta de dos fases de validación y operacional. La primera fase, se la conoce como IOV (In Orbit Validation). Para esta, se utilizaron los satélites GIOVE-A y GIOVE-B, que fueron lanzados en el 2005 y en 2008, respectivamente. Además de esto, en el año 2011, se lanzaron 2 satélites que iban a conformar una mini constelación de cuatro satélites, los 2 satélites restantes se lanzaron en el 2012, totalmente operativos 
y con su correspondiente infraestructura terrestre. Y la segunda fase, se la conoce como FOC (Full Operational Capability), que incluye una fase intermedia con 18 satélites operativos (4 de la fase de validación IOV y otros 14 satélites). El sistema de navegación se completará con 30 satélites, sus respectivos centros de control se ubicarán en Europa, además de una red de estaciones permanentes que estarán distribuidas por todo el mundo.

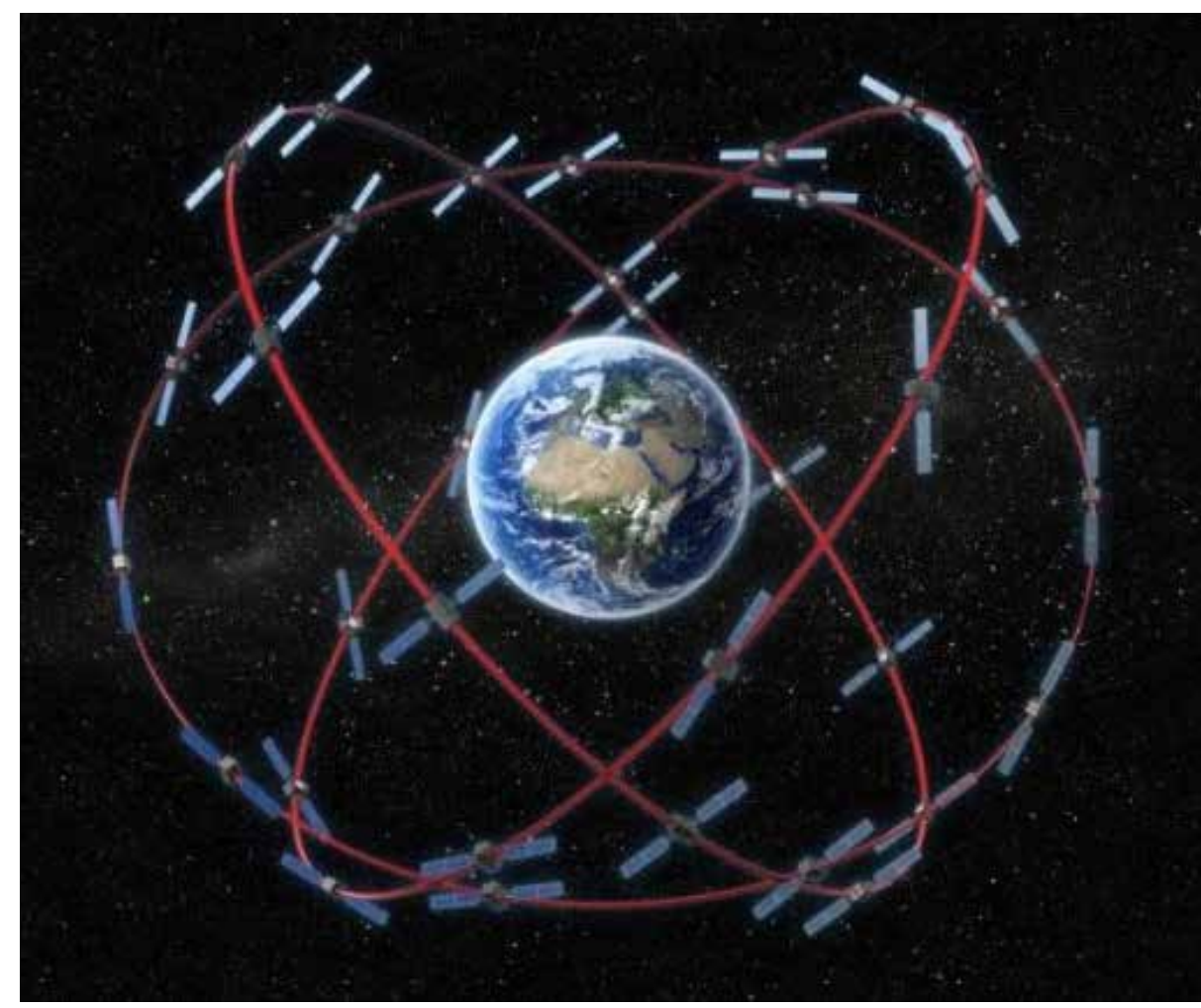

Figura 1.- Constelación Galileo

Fuente: (Martínez, 2013)

\section{SEGMENTOS DEL SISTEMA GALILEO}

El Sistema Galileo está conformado por 3 segmentos: el espacial, el terrestre y el de usuario. El Segmento Espacial, consiste en 30 satélites, de los cuales 27 son operativos y 3 de reserva, ubicados en 3 órbitas aproximadamente a $23222 \mathrm{~km}$ de altitud con respecto al geocentro. Cada plano orbital tiene una inclinación de 56 grados con respecto al Ecuador de referencia, el período orbital demorará cerca de 14 horas 21.6 minutos solares (Katsigianni, 2018), esta elección asegura que el ciclo del trayecto de cada satélite se repita cada 17 órbitas o lo que equivale a 10 días (Bartolomé, et al., 2015).

El Segmento Terrestre de Galileo, consta de un Centro de Control (GCC) ubicado en territorio perteneciente a la Unión Europea, este se encarga del control de los satélites 
y del cálculo de los distintos mensajes de navegación para enviar a los satélites. La Red Global de Sensores Galileo (GSS), toman las observaciones de los satélites, estas se envían al Centro de Control de Galileo mediante una red que facilita la comunicación. El centro de control sincroniza las distintas señales de tiempo de todos los satélites de galileo con los osciladores de la tierra. Las estaciones de unión son las encargadas del intercambio de información entre los satélites y los centros de control terrestre.

Los servicios que ofrece el sistema Galileo son el Open Service, Safety Of Life, Commercial Service, Public Regulated Service, Search and Public Rescue Service (Bartolomé, et al., 2015), que se detallan a continuación:

Open Service (OS): Servicio abierto sin cargo para cualquier usuario que posea un equipo de navegación compatible con Galileo. Estará provisto en las bandas E1 y E5, además será comparable con el servicio que ofrece GPS en sus señales civiles L1C/A, L2C o L5. Este servicio, transmitirá el mensaje F/NAV (Free accesible Navigation message).

Safety Of Life (SOL): Seguridad de la vida, este servicio mejora las prestaciones del Open Service incluyendo la provisión de la integridad del sistema mundial. Además, transmitirá el mensaje I/NAV (Integrity Navigation message).

Commercial Service (CS): Servicio comercial, la información que se deriva de este posee una mayor precisión para motivo de posicionamiento o navegación, es decir, que brinda un valor agregado a los datos con respecto al Open Service. Una ventaja que presenta CS con respecto a cualquier otro sistema de GNSS, es la capacidad de transmitir globalmente datos en tiempo real mediante el uso de la banda E6. Y, transmitirá el mensaje I/NAV (Integrity Navigation message) y el C/NAV (Commercial Navitation message).

Public Regulated Service (PRS): El Servicio público regulado, dará servicio de posicionamiento a los usuarios que requieran un alta continuidad, el acceso será restringido únicamente para usuarios gubernamentales autorizados. La política de control de acceso está implementada mediante la encriptación de la señal PRS y la administración de las claves de descifrado. El mensaje transmitido por este servicio es el G/NAV (Governammental Navigation message).

Search and Public Rescue Service (SAR): Servicio público de búsqueda y rescate, a nivel mundial, se encargará de transmitir mensajes de alerta que serán recibidos en tiempo real, este servicio ofrecerá el apoyo en varias operaciones de riesgo.

En la siguiente figura 2, se detallan las frecuencias y las bandas en las que se transmiten los diferentes servicios de Galileo, además, se expresa la compatibilidad del sistema con GPS. 


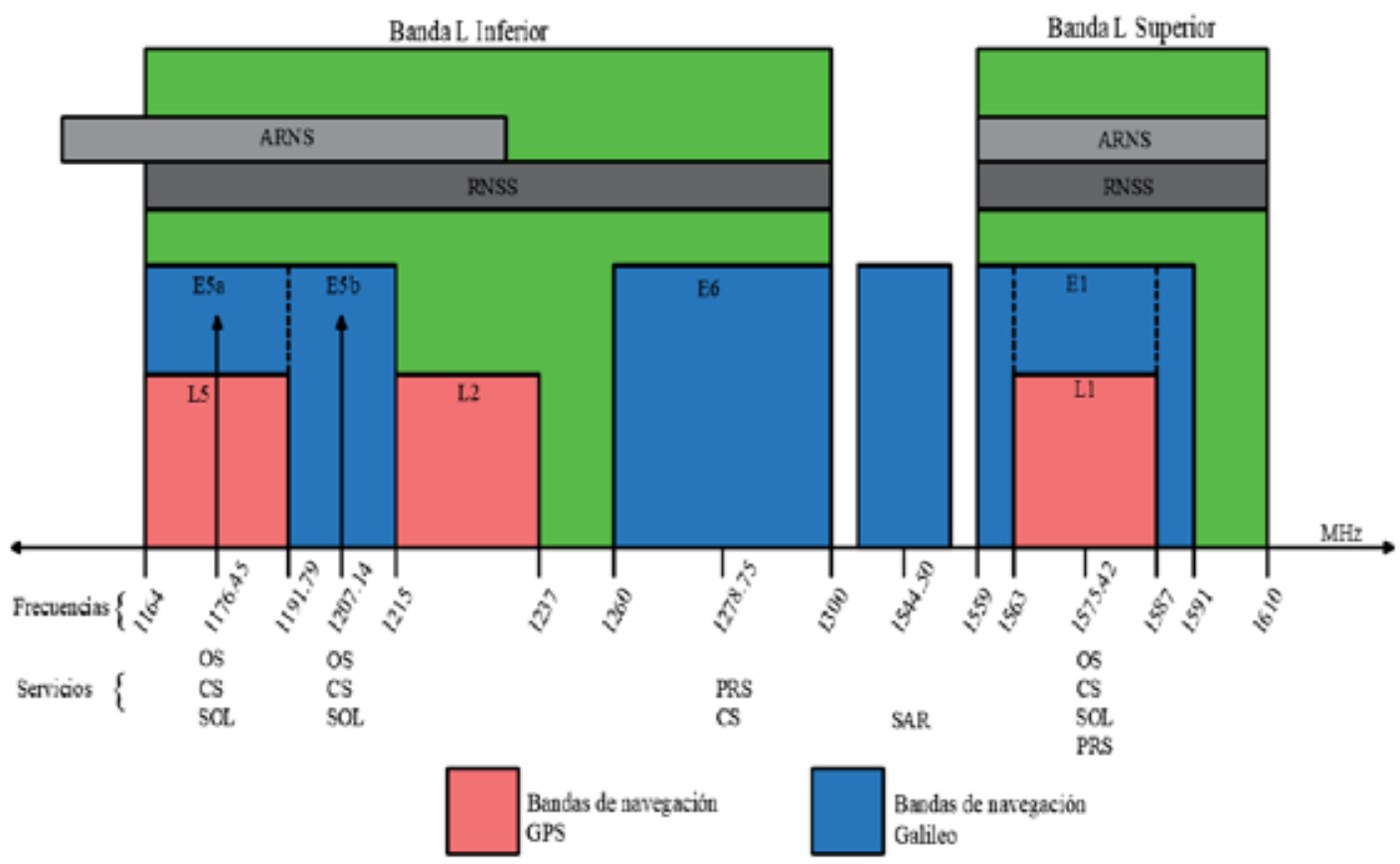

Figura 2.- Frecuencias y bandas de los Servicios de Galileo

Fuente: (Martínez, 2013) adaptado por el autor

\section{METODOLOGÍA}

Para obtener datos del Sistema Galileo, se seleccionaron 3 bases (Tabla 1) pertenecientes a la Red GNSS de Monitoreo Continuo del Ecuador (REGME) y enlazadas a la red SIRGAS - CON. El rastreo de esta constelación se realizó por un período que abarca desde el día 200 (semana GPS 1958) hasta el día 224 (semana GPS 1961), año 2017. La ubicación de estas estaciones, se muestran en la figura 3.

Tabla 1.- Estaciones de Monitoreo Continuo

\begin{tabular}{|c|c|}
\hline Nombre de la Estación & Ubicación (Provincia) \\
\hline BHEC & Los Ríos (Costa) \\
\hline CXEC & Cotopaxi (Sierra) \\
\hline EPEC & Pichincha (Sierra) \\
\hline
\end{tabular}




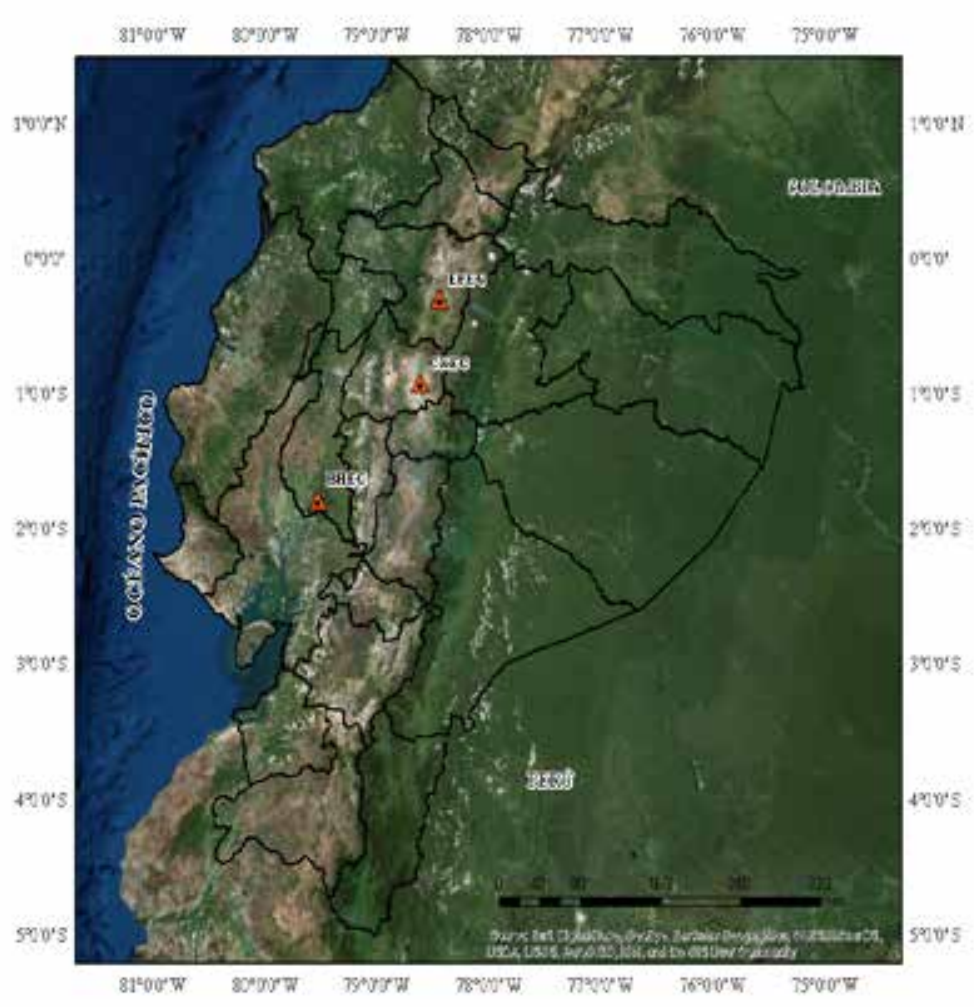

Figura 3.- Mapa de ubicación del Proyecto

Los equipos utilizados para el rastreo de señal de GALILEO corresponden a las especificaciones que se muestran en la Tabla 2:

Tabla 2.- Descripción de equipos GNSS

\begin{tabular}{|c|c|}
\hline Descripción & Especificación \\
\hline Tipo de Receptor & TRIMBLE NETR9 \\
\hline Modelo de Antena & GNSS CHOKE w/SCIS Dome \\
\hline Sistema Satelital & GPS + GLONASS + GALILEO \\
\hline Intervalo de Registro & 1 Segundo \\
\hline Ángulo de máscara & Orados \\
\hline Formato de Archivo & Origin TRIMBLE (T02) \\
\hline
\end{tabular}

Fuente: http://www.geoportaligm.gob.ec

Los datos crudos (nombre.t02), obtenidos a partir del rastreo de cada estación de monitoreo continuo (EMC), que contienen la información de las tres constelaciones satelitales (GPS, GLONASS y Galileo), por el período de 25 días, se transformaron para obtener los archivos RINEX con sus diferentes extensiones, como se muestra en la siguiente tabla 3: 
Tabla 3.- Extensiones de los archivos RINEX.

\begin{tabular}{|c|c|}
\hline Detalle & Extensión \\
\hline Archivo de observación & .\#\# \\
\hline Archivo de navegación GPS: &.$\# \# \mathrm{n}$ \\
\hline Archivo de navegación GLONASS: &.$\# \# \mathrm{~g}$ \\
\hline Archivo de navegación GALILEO: &.$\# \# 1$ \\
\hline Archivo de información meteorológica: &.$\# \# \mathrm{~m}$ \\
\hline
\end{tabular}

Fuente: https://cddis.nasa.gov

El procesamiento de datos se realizó mediante el uso del software RTKLIB versión 2.4.2, que se puede descargar en: www.rtklib.com, con su aplicación RTKPOST, donde los insumos que se utilizaron fueron los archivos de observación (.\#\#) y los archivos de navegación de GALILEO (.\#\#l), la interfaz de procesamiento se puede observar en la siguiente figura 4.

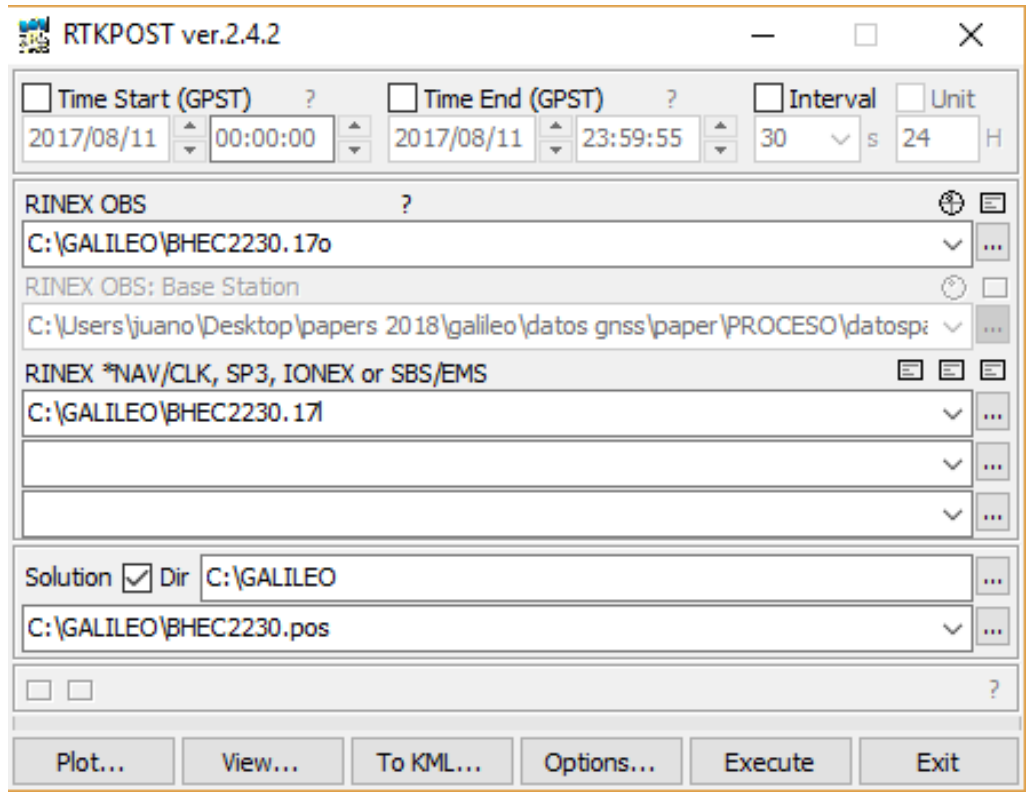

Figura 4.- Interfaz aplicación RTKPOST

Para determinar las coordenadas geodésicas (latitud, longitud y altura elipsoidal), se configuró el software para que utilice PPP estático como método de posicionamiento, la elevación de la máscara de 15 grados, efemérides transmitidas de los satélites, y únicamente la constelación GALILEO, como se muestra en la figura 5. 


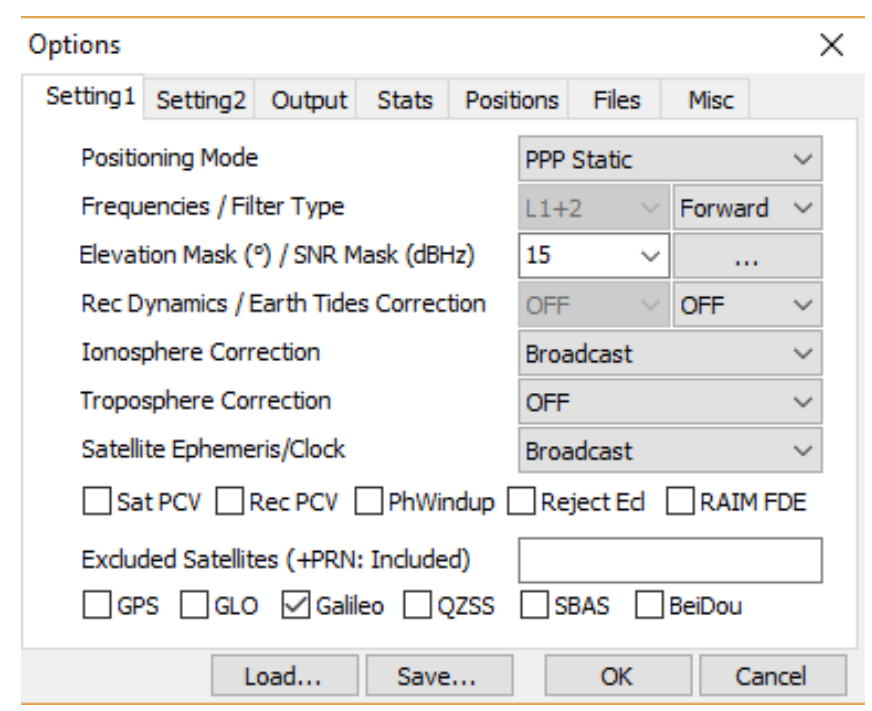

Figura 5.- Configuraciones para el procesamiento

El proceso consistió en varias etapas, en la primera se procesaron los datos de las 3 estaciones de monitoreo de todos los días, identificando las soluciones (coordenadas). Si no existía solución, se descartaba el día respectivamente de la estación. Una vez identificados los días que efectivamente presentaban soluciones, estos se procesaron por bloques. En la tabla 4, se muestra los intervalos e incrementos de cada bloque:

Tabla 4.- Bloques de tiempo para procesamiento

\begin{tabular}{|c|c|c|}
\hline Bloque & Lapsos de tiempo & Incremento \\
\hline 1 & 1 segundos $-10 \mathrm{~min}$ & 30 segundos \\
\hline 2 & $10 \mathrm{~min}-30 \mathrm{~min}$ & 1 minuto \\
\hline 3 & $30 \mathrm{~min}-1 \mathrm{~h} 30 \mathrm{~min}$ & 10 minutos \\
\hline 4 & 2 horas -24 horas & 1 hora \\
\hline
\end{tabular}

Se construyó el bloque 1 con el lapso de 10 minutos, para asemejar a los diferentes tipos de posicionamiento cinemático como stop and go y continuo. El bloque número 2, se construyó con tiempos desde 10 minutos hasta 30 minutos, estos lapsos de tiempo asemejan un posicionamiento estático rápido. El bloque 3, tiene su lapso entre 30 minutos hasta 1.5 horas, este tiempo, en comparación, es similar al utilizado por el método estático, que dependiendo de la base se utiliza para alcanzar una precisión a nivel centimétrico. El último bloque, tuvo sus lapsos de 2 horas hasta 24 , este brinda una muy buena precisión ya que puede alcanzar el nivel centimétrico, utilizando un método relativo. 
El procesamiento por bloques se realizó en 2 fases correspondiente a intervalos de procesamiento 30 y 1 segundo. En la primera, si se verificaba la variación de datos entre lapsos se procedía a la siguiente fase (intervalo de 1 segundo), caso contrario se descartaba el día. Una vez procesados todos los datos, y obtenidas las coordenadas geodésicas, se transformaron a UTM. A partir de éstas, se calculó la distancia horizontal entre las coordenadas determinadas con Galileo mediante la técnica PPP estático y las soluciones semanales SIRGAS. Esta distancia entre coordenadas indica el error en posición horizontal que presenta la constelación Galileo las 3 EMC.

\section{RESULTADOS Y ANÁLISIS}

Del análisis realizado, las estaciones presentan buenos resultados ante la recepción de datos de Galileo, BHEC (día 223), CXEC (día 219) y EPEC (día 213). A continuación, se muestran los gráficos de estas estaciones, donde, el eje de las ordenadas representa el error de las coordenadas determinadas únicamente con Galileo con respecto a las soluciones semanales SIRGAS; y el eje de las abscisas representa los lapsos de tiempo. En la figura 6, se muestra la gráfica error distancia horizontal vs tiempo que se presentaron durante el rastreo Galileo de la estación BHEC durante el día 223 (24 horas). Donde la línea azul corresponde a los datos a 30 segundos, y la línea roja corresponde a 1 segundo de intervalo de procesamiento.

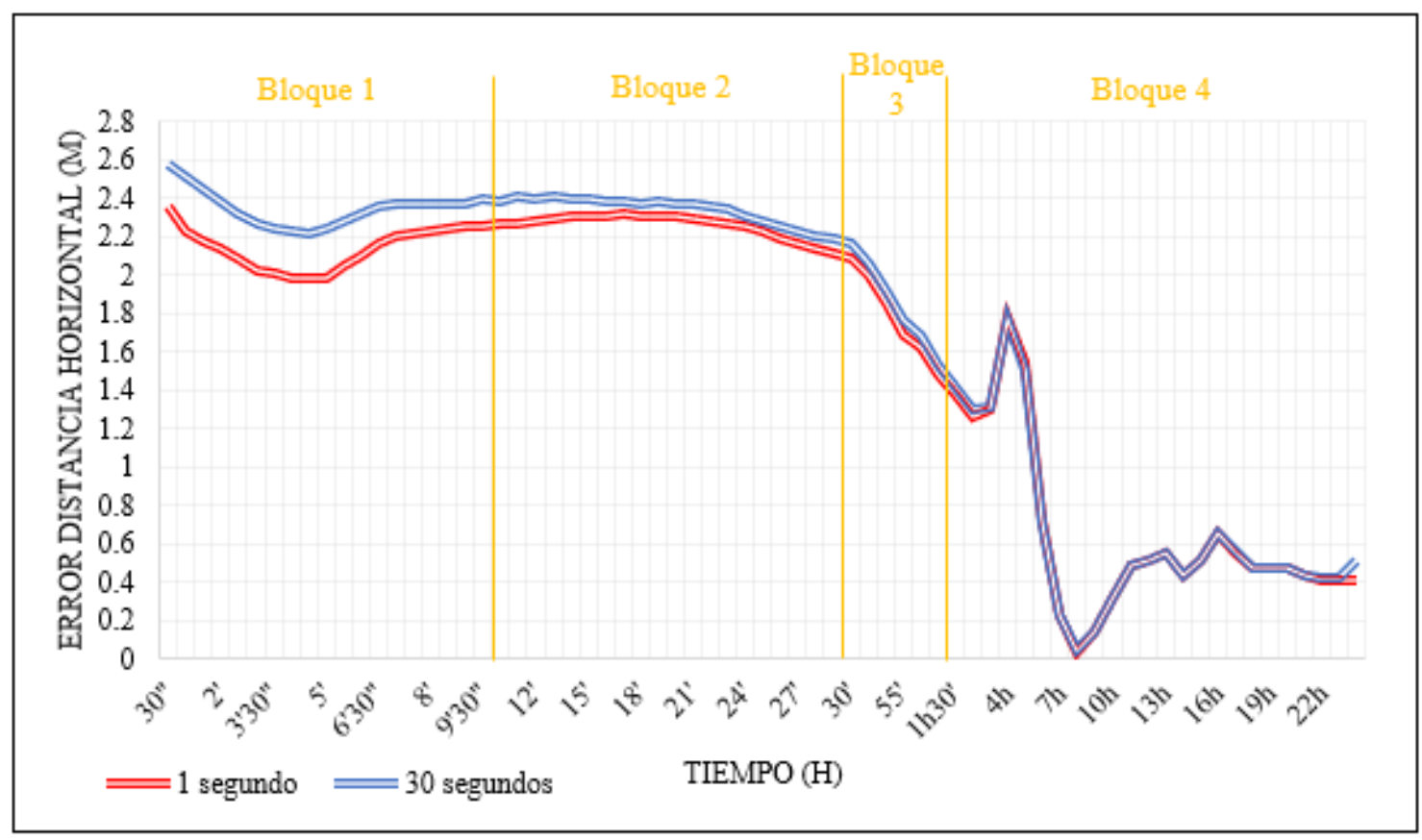

Figura 6.- Error en posición, estación BHEC

Como se muestra en la figura 6, el bloque 4 que abarca desde 1 hora 30 minutos hasta 24 horas, es el que tiene menor error, llegando incluso hasta los $5 \mathrm{~cm}$, cabe considerar que 
para las 4 horas, existe un pico (variación significativa), esto probablemente se debe a la geometría de los satélites en ese tiempo; el bloque 3 tiene su lapso entre 30 minutos y 1 hora 30 minutos, y presenta errores entre 2 metros y 1.40 metros, debido al tiempo que ocupan estos, no es aplicable la técnica PPP ya que para estos tiempos es recomendable un posicionamiento relativo. Además, se aprecia que las diferencias entre intervalos de procesamiento de 1 y 30 segundos son despreciables.

Por otro lado, el bloque 1, el mayor error de entre $2.6 \mathrm{~m}$ y $2.2 \mathrm{~m}$ corresponde al procesamiento a intervalo de 30 segundos, mientras que el procesamiento a 1 segundo tiene su error entre $2.4 \mathrm{~m}$ y $2 \mathrm{~m}$. Finalmente, en el bloque 2 , se aprecia que la diferencia entre observaciones procesadas con intervalos de 1 y 30 segundos varía por unos pocos centímetros y el error en este lapso corresponde a un promedio de 2.3 metros.

En la figura 7, se puede apreciar la gráfica del error en posición correspondiente a la estación CXEC. El rastreo Galileo, se llevó a cabo durante las 24 horas del día 219, con intervalos de procesamiento de 1 (línea roja) y 30 segundos (línea azul).

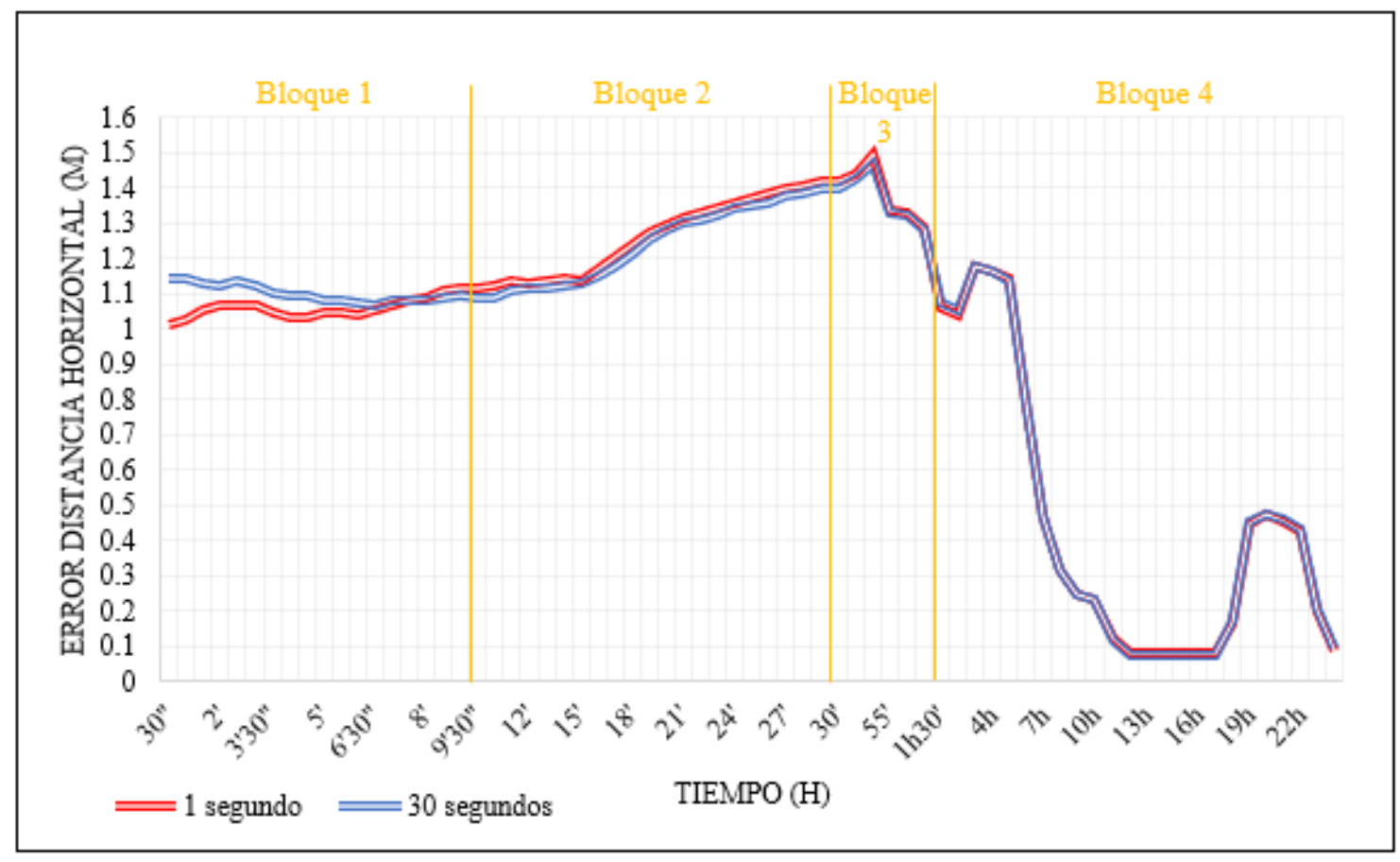

Figura 7.- Error en posición, estación CXEC

Como se muestra en la figura 7 , el bloque 4, presenta un error mayor que sobrepasa el metro, y el menor error llega hasta 10 centímetros. El bloque 3, muestra errores superiores a 1 metro. El procesamiento con 1 y 30 segundos para los dos bloques es similar y no presentan diferencias significativas. 
El bloque 1, presenta un error medio de 1.05 metros, se puede apreciar que al inicio de la sesión, hay una diferencia de 10 centímetros entre los errores a 1 y 30 segundos, pero esta se vuelve despreciable a los 8 minutos de rastreo. Finalmente, el bloque 2 presenta errores desde $1.1 \mathrm{~m}$ que se incrementa hasta $1.4 \mathrm{~m}$.

En la figura 8, se muestran los errores correspondientes a la estación EPEC, durante el día 219 (24 horas).

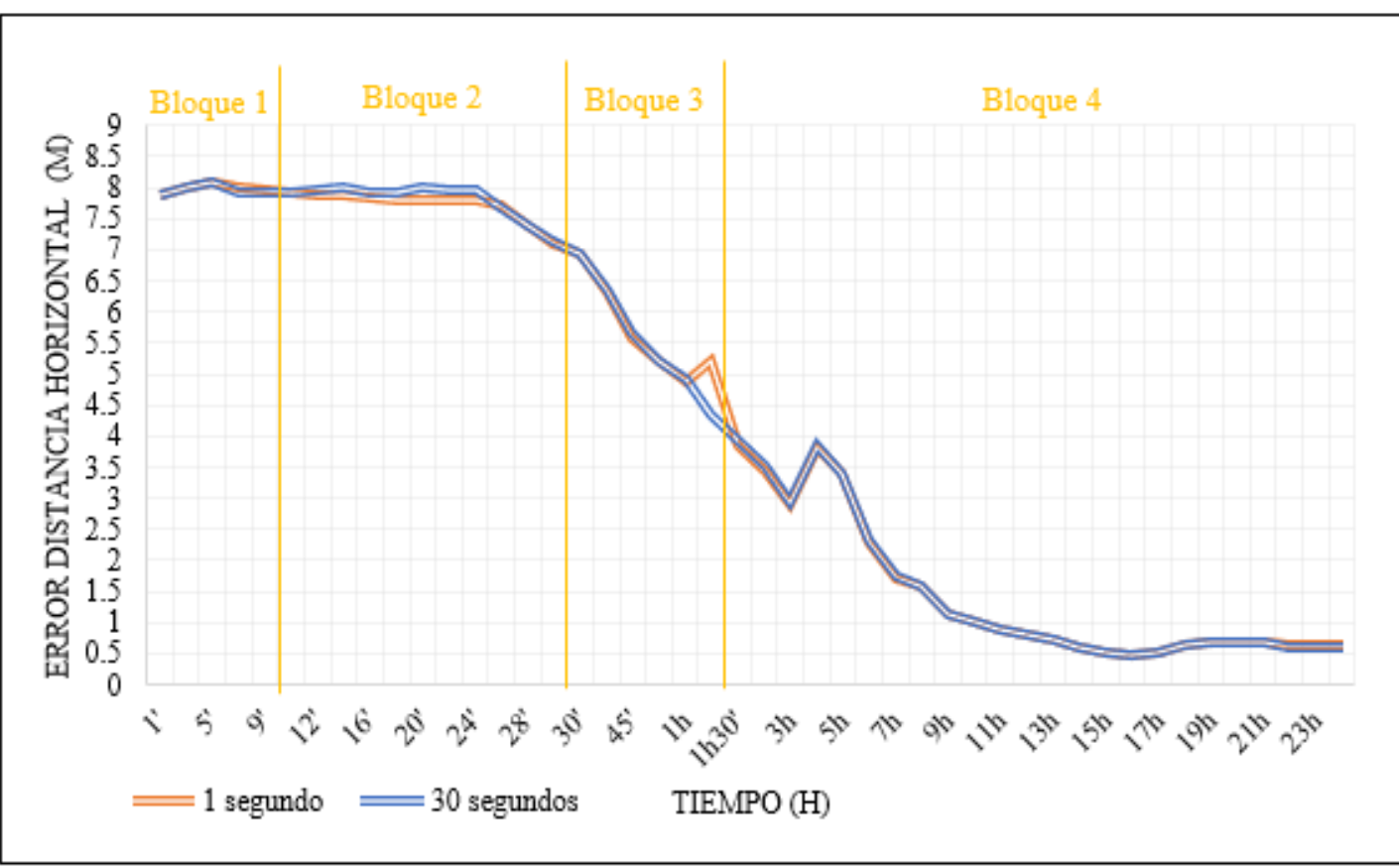

Figura 8.- Error en posición, estación EPEC

Como se muestra en la figura 8 , el bloque 4 presenta un error que está entre los 4 metros hasta 50 centímetros. El bloque 3, presenta un error que va desde 7 metros hasta 4 . El bloque 1 posee un error de 8 metros que se mantiene por el lapso de tiempo. Finalmente, el bloque 2, presenta un error de 8 metros y disminuye hasta los 7 metros. Esta estación presenta un problema, ya que a comparación de las estaciones anteriores (CXEC y BHEC) presenta un error mucho más grande.

Utilizando las estaciones BHEC y CXEC (véase figura 6 y 7) durante los primeros 30 minutos de rastreo se puede conseguir un error medio de 1.5 metros, que es aplicable para diferentes actividades como la navegación o la georreferenciación de cartografía a escalas menores que 1:5000.

En vista de estos resultados, el estudio de la constelación Galileo sobre el Ecuador debe continuar, se debe analizar los errores en posición a partir de este sistema cuando se haya 
completado el segmento espacial. Posteriormente, se debe realizar un análisis empleando 3 constelaciones de GNSS: GPS, GLONASS y Galileo, a partir de éstas se debe analizar el error en posición mediante la utilización de un software científico como Bernese o Gamit Globk.

\section{CONCLUSIONES}

El intervalo de procesamiento de 1 y 30 segundos es similar para ambos casos, únicamente una variación considerable se puede apreciar para la estación CXEC, donde las diferencias son de aproximadamente 20 centímetros.

De los bloques utilizados para el procesamiento, el que presenta un menor error es el bloque 4 , donde se pueden obtener errores a nivel inferior a los decímetros.

Los bloques 1 y 2, son los que presentan un mayor error, para la estación BHEC un error a nivel de los 2 metros, y para la estación CXEC de 1 metro. La estación EPEC, presenta un error más alto que las otras dos estaciones, aproximadamente 8 metros, esto puede deberse a la ubicación geográfica, la geometría de los satélites y a posibles pérdidas de señal.

\section{AGRADECIMIENTOS}

Un agradecimiento especial al Instituto Geográfico Militar de Ecuador por facilitar los datos para el desarrollo de esta investigación.

\section{REFERENCIAS}

Agencia Espacial Europea, ESA. (2017). Galileo Navigation. Obtenido de http://www.esa.int/.../ What_is_Galileo. Recuperado el 20 de agosto de 2018.

Agencia Espacial Europea, ESA. (2018). Un Nuevo Lanzamiento Amplía el Alcance Global de Galileo. Obtenido de: http://www.esa.int/. Recuperado el 20 de agosto de 2018.

Albán, M., Romero, R., Tierra, A. (2017). Cálculo de coordenadas usando el método Precise Point Positioning - PPP estático mediante el software RTKLIB. Geociencia \& Geodatos, $63-69$.

Bartolomé, J. P., Maufroid, X., Hernández, I. F., Salcedo, J. A. L., \& Granados, G. S. (2015). Overview of Galileo system. In GALILEO Positioning Technology (pp. 9-33). Springer, Dordrecht.

Cai, C., Gao, Y., Pan, L., \& Zhu, J. (2015). Precise point positioning with quad-constellations: GPS, BeiDou, GLONASS and Galileo. Advances in space research, 56(1), 133-143.

Cogo, J., Garcia, J. G., Roncagliolo, P. A., \& Muravchik, C. H. (2012). GNSS multi-constellation positioning problem: A numerical optimization approach.

Comisión Europea en España. (2016). Comunicado de prensa: "GALILEO entra en funcionamiento".

Gendt, G., Altamimi, Z., Dach, R., Söhne, W., \& Springer, T. (2011). GGSP: realisation and maintenance of the Galileo terrestrial reference frame. Advances in Space Research, 47(2), 
174-185.

Huerta, E., Mangiaterra, A., \& Noguera, G. (2005). GPS, Posicionamiento Satelital. Rosario: UNR Editora.

Katsigianni, G., Loyer, S., Perosanz, F., Mercier, F., Zajdel, R., \& Sośnica, K. (2018). Improving Galileo orbit determination using zero-difference ambiguity fixing in a Multi-GNSS processing. Advances in Space Research.

Li, X. et al. (2015). Precise positioning with current multi-constellation Global Navigation Satellite Systems: GPS, GLONASS, Galileo and BeiDou. Sci. Rep. 5,8328; DOI:10.1038/ srep08328

Martínez, J. (2013). Sistema Galileo: El concepto europeo de la navegación por satélite. Universidad Politécnica de Catalunya.

Montenbruck, O., Steigenberger, P., Prange, L., Deng, Z., Zhao, Q., Perosanz, F., ... \& Schmid, R. (2017). The Multi-GNSS Experiment (MGEX) of the International GNSS Service (IGS)achievements, prospects and challenges. Advances in space research, 59(7), 1671-1697.

Ventura-Traveset, (2016). La Navegación por satélite y el Sistema Europeo Galileo. Agencia Espacial Europea, ESA. 